\title{
Examining Medicaid Expansion and the Treatment of Substance Use Disorders
}

\author{
By Jevay Grooms and Alberto OrtegA*
}

Since the year 2000, fatal drug overdoses have increased by 265 percent (National Safety Council Statistics Department 2018). In 2014, 21.5 million Americans had a substance use disorder (SUD) and 7.9 million had a co-occurrence of a mental health condition (Substance Abuse and Mental Health Services Administration 2016). Yet, historically, healthcare services for SUDs have not been treated in the same manner as other health conditions (Buck 2011), with treatment for SUDs not covered by some private insurance. Ensuring individuals who suffer from SUDs have access to proper treatment is essential not only for immediate care but for the care of their chronic condition and the myriad of comorbidities associated with their condition, which can span far beyond the discontinued use of substances. The National Institute of Drug Abuse (2016) refers to addiction as a chronic but treatable disease with rates of relapse similar to those of other illnesses with a behavioral component (e.g., diabetes).

In 2010, the Obama Administration passed the Patient Protection and Affordable Care Act (ACA) which expanded health insurance coverage and services offered to qualified recipients. A provision of the ACA is its consideration of

\footnotetext{
* Grooms: Department of Economics, Howard University, ASB-B Room 307, 2400 6th Street NW, Washington, DC 20059 (email: jevay.grooms@howard.edu); Ortega: Whitman College, 345 Boyer Avenue, Walla Walla, WA (email: ortegaa@whitman.edu). We are grateful for extensive comments from Anirban Basu and Alice Ellyson as well as helpful comments from Kosali Simon and members of the Health Workshop at the School of Public and Environmental Affairs: Indiana University and DITE Cohort 10 for all their comments at the Spring Workshop 2018.

${ }^{\dagger}$ Go to https://doi.org/10.1257/pandp.20191090 to visit the article page for additional materials and author disclosure statement $(\mathrm{s})$.
}

SUD services as an essential health benefit. ${ }^{\text {T }}$ A vital aspect of the ACA and the focus of this paper is the expansion of Medicaid. In addition to increases in services offered through the ACA, the expansion of Medicaid substantially altered the eligibility requirements in two ways: (i) increasing the household income criteria from 100 percent of the federal poverty line to 138 percent; and (ii) covering adults without dependent children. Uninsurance rates are the highest among young adults and it is believed that this population also has higher health needs. Simon, Soni, and Cawley (2017) find that the Medicaid expansion led to increases in health insurance coverage among low-income childless adults. Furthermore, childless adults have been found to have more substance abuse and mental health visits relative to their counterparts with children (Haber, Khatutsky, and Mitchell 2000). Additionally, the National Survey on Drug Use and Health (2010-2013) find that roughly 37 percent of respondents cited lack of health coverage as their reason for not receiving treatment (Substance Abuse and Mental Health Services Administration 2014).

In this paper, we investigate whether the supply of SUD treatment responds to Medicaid expansion, via the ACA. We complement existing research on the effects of Medicaid expansion on SUD treatment admissions. These findings are both paramount in understanding the treatment of a vulnerable population and also have implications in addressing the social externalities associated with SUDs.

\section{Medicaid Expansion and SUDs}

Although there has been a considerable amount of research on the effects of Medicaid

\footnotetext{
${ }^{1}$ Essential Health Benefits Requirements, 42 U.S.C. $\S 18022$.
} 
expansion, the evidence on the role of the ACA on SUD treatment is scarce. Wen et al. (2017) finds that Medicaid expansion under the ACA led to an increase in physician prescriptions to treat opioid use disorders. Maclean and Saloner (forthcoming) find an increase in both Medicaid-reimbursed prescriptions as well as substance abuse treatment admissions which were paid via Medicaid insurance, post-expansion. Similarly, Hamersma and Maclean (2018) suggest that private and public insurance expansions are associated with a greater number of treatment admissions among children ages 12 to 18 . Conversely, when examining the ACA dependent coverage provision, which mandated health insurers to offer coverage to adult children of their beneficiaries under age 26, Saloner et al. (2018) finds that the provision did not necessarily increase the demand for SUD treatment among those privately insured.

Grooms and Ortega (2018) add to this literature on ACA and Medicaid expansion by examining the type of insurance recorded at admission to offer insights into the access of SUD treatment and subsequent healthcare. Maclean and Saloner (forthcoming) find an increase of 57 percent in admissions paid by Medicaid insurance in 2014. However, given that many states expanded Medicaid as part of the ACA after 2014, this paper is limited in the number of post-expansion years. Grooms and Ortega (2018) find that on average the effect increases admissions with Medicaid insurance by 85 percent.

In this paper, we investigate a commonly overlooked component within this literaturethe effects on the supply or efficacy of care. Independent of the ACA, treatment facilities have been found to have difficulty in finding open slots for patients (Jones et al. 2015). Acknowledging that treatment facilities face capacity constraints, we aim to complement the aforementioned literature by offering some basic insight into the supply of treatment facilities. Thus, we examine the effect of ACA expansion on the number of treatment facilities as well as an imperfect proxy for the efficacy of care- the number of days waited before admission. The latter measure is important given that a longer waiting period, prior to admission, has been found to decrease the likelihood of treatment retention (Claus and Kindleberger 2002).

\section{Data and Empirical Strategy}

Our data is collected from the National Survey of Substance Abuse Treatment Services (N-SSATS) dataset as well as the Treatment Episode Data Set-Admissions (TEDS), made available by The Substance Abuse and Mental Health Services Administration. ${ }^{2}$ The full N-SSATS dataset is comprised of 97,000 facilities (both in-patient and out-patient), from 2010 to $2016 .{ }^{3}$ It is important to note that the analysis presented in this paper is intended to complement studies (Grooms and Ortega 2018 and Maclean and Saloner forthcoming) which utilize TEDS. This dataset is a national census of admissions into SUD treatment facilities and accounts for roughly two-thirds of the entire population of treatment admissions (Dave and Mukerjee 2011). We include state demographics from the University of Kentucky Center for Poverty Research (UKCPR). 4

In this study, we compare states that expanded Medicaid on January 1, 2014 to those that did not. ${ }^{5}$ Our empirical strategy is similar to that of Grooms and Ortega (2018) which includes leads and lags within a difference-in-differences (DID) model, allowing us to identify any dynamic effects resulting from the policy over time (i.e., event-study). In this analysis our outcome variable is the total number of facilities, number of facilities which accept Medicaid, and the number of days waited prior to receiving clinical services. ${ }^{6}$

Grooms and Ortega (2018) find an increase in the number of admissions with Medicaid insurance post-expansion. Figure 1 reports their

\footnotetext{
${ }^{2}$ https://www.samhsa.gov/.

${ }^{3}$ Roughly 22 percent of the facilities surveyed are not included in the final dataset due to incomplete information. Our final N-SSATS dataset is comprised of 68,368 facilities across 32 states.

${ }^{4}$ More information can be found at http://ukcpr.org/ resources/national-welfare-data.

${ }^{5}$ The expansion states in our sample are Arizona, Arkansas, Colorado, Delaware, Hawaii, Illinois, Iowa, Kentucky, Maryland, Massachusetts, Nevada, New Mexico, North Dakota, Oregon, and West Virginia. Non-expansion states are Alabama, Florida, Georgia, Idaho, Kansas, Maine, Mississippi, South Dakota, Tennessee, Texas, Utah, and Wyoming, states who as of December 31, 2015, have not expanded Medicaid in any form.

${ }^{6}$ The analysis includes state demographics as well at state and year fixed effects, and clustered standard errors at the level of policy implementation.
} 
event-study estimates by insurance type from 2010 to 2016. Estimates for post-treatment years, 2014 to 2016, are statistically insignificant for admissions with private insurance and no insurance. This result holds for individuals with Medicare/Tricare insurance and also for those who did not report any insurance type. The former is expected given that this population is least likely to be affected by the Medicaid expansion. Post-treatment coefficients associated with Medicaid insurance are statistically significant and increasing in magnitude from the implementation period. They find that, on average, the expansion of coverage and benefits resulted in an 85 percent increase in admissions with Medicaid insurance into SUD treatment facilities. This result serves as the impetus for examining supply-side effects resulting from ACA expansion.

\section{Results}

Given Figure 1, we begin the investigation of the supply of treatment facilities by examining trends from 2010 to 2016. Figure 2 indicates that expansion and non-expansion states seem to have similar trends in the number of facilities pre- and post-policy implementation. To further investigate, we employ the aforementioned DID model. The findings reveal no difference in the number of overall facilities as a result of Medicaid expansion. This also holds true for facilities that accept Medicaid as a form of insurance. Ideally, we would be able to speak to the composition and capacity of individual facilities; unfortunately, due to data limitations we are unable to.

Our proxy for availability of care is the amount of time an individual waits before entering treatment. Since we find no change in the number of facilities, one can surmise that a large increase in the number of individuals being admitted may adversely affect treatment via increased wait times. The same identification strategy reveals no difference in wait times post-expansion across insurance types.

Since Grooms and Ortega (2018) find an overall increase in the number of admissions in expansion states relative to non-expansion states, our results may indicate that facilities have been adequately prepared to provide care to the influx of new admissions - at least for the first few years post Medicaid expansion.
Panel A. Medicaid

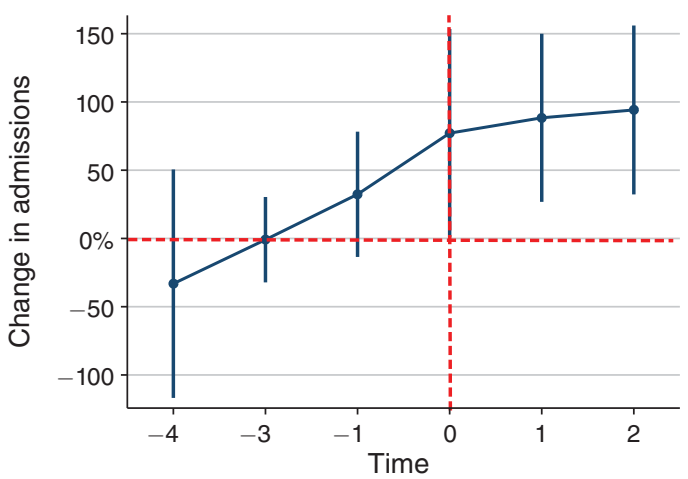

Panel B. No insurance

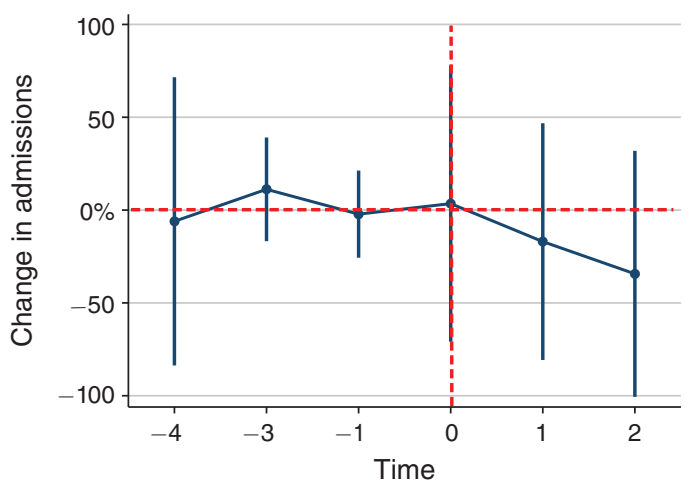

Panel C. Private insurance

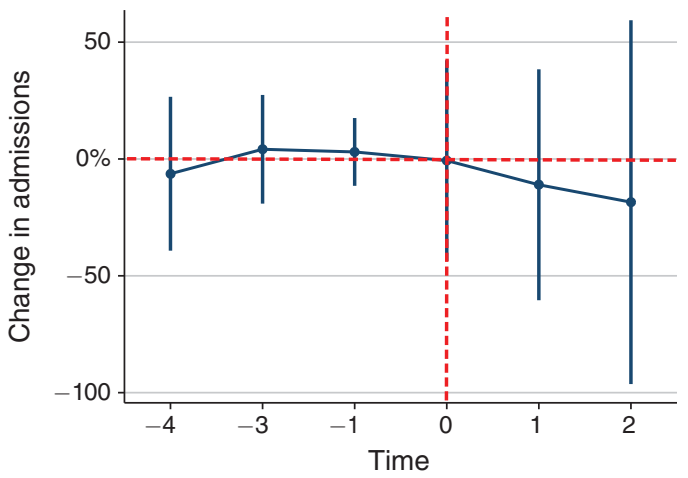

Figure 1. Event Study for Admissions by Insurance Type (GROOMS AND ORTEGA 2018)

Notes: All analysis is relative to 2012 and $t=0$ at year 2014 . Also while Medicare and Tricare are not the same type of insurance, that is the manner in which intake admissions are coded.

We test the robustness and sensitivity of our results by estimating alternative specifications, functional forms, and potentially confounding policies. To assure our results are not driven 
Panel A. All facilities

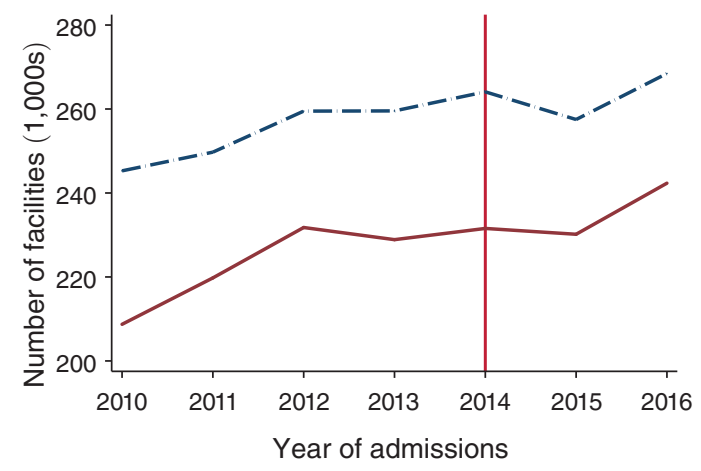

Panel B. Medicaid facilities

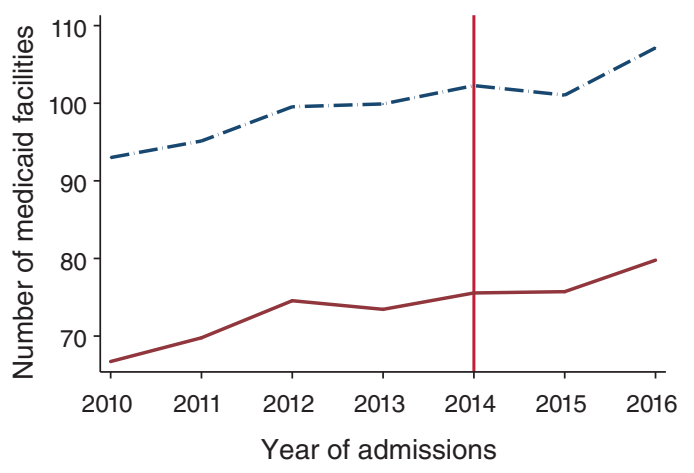

$$
\begin{aligned}
& -\cdots \text { Expansion states } \\
& \text { Non-expansion states }
\end{aligned}
$$

Figure 2. Trends in Average Number of Substance Use DisORDER FACILITIES

by confounding public policies, we control for other interventions that may affect substance abuse admissions. During the time of our study, some states implemented prescription drug monitoring programs (PDMPs) in order to facilitate the tracking of prescription drugs. PDMPs have been found to affect drug prescribing, drug misuse, as well as admissions into substance abuse facilities.' We also consider states that have passed pain clinic laws during the time of our study. In an attempt to mitigate the opioid epidemic, these laws can alter drug use via substitution effects resulting from a change in the opportunity cost of opioid consumption.

\footnotetext{
${ }^{7}$ Buchmueller and Carey (2018); Dave, Grecu, and Saffer (2017); Ellyson, Grooms, and Ortega (2017).
}

Controlling for these policies do not affect our findings.

\section{Concluding Remarks}

In previous decades SUDs were not considered a disease which needed extensive treatment, rather, they were considered a choice intertwined with an individual's moral fabric. As we continue to better understand how drug use impacts brain chemistry, it is imperative that we investigate the role adequate access to quality care can play in the long-term treatment of the disease, comorbidities, and social externalities. Our results indicate that while there was a substantial increase in SUD treatment admissions with Medicaid coverage, there is no effect of Medicaid expansion on the supply of facilities or the time patients waited before clinical services were rendered.

Yet to be fully addressed by the literature is explicitly examining the efficacy of care. It is imperative to understand the type of care provided, the quality of care, and possible heterogeneous effects across race, ethnicity, and gender. Another looming concern is the understanding of the co-occurrence of SUDs and mental health conditions. Antwi, Moriya, and Simon (2015) finds the dependent coverage provision of the ACA to be associated with an increase in mental health-related visits; however, it is still unclear how facilities are treating both ailments simultaneously to ensure adequate care.

\section{REFERENCES}

Antwi, Yaa Akosa, Asako S. Moriya, and Kosali I. Simon. 2015. "Access to Health Insurance and the Use of Inpatient Medical Care: Evidence from the Affordable Care Act Young Adult Mandate." Journal of Health Economics 39: 171-87.

-Buchmueller, Thomas C., and Colleen Carey. 2018. "The Effect of Prescription Drug Monitoring Programs on Opioid Utilization in Medicare." American Economic Journal: Economic Policy 10 (1): 77-112.

Buck, Jeffery A. 2011. "The Looming Expansion And Transformation Of Public Substance Abuse Treatment Under The Affordable Care Act." Health Affairs 30 (8): 1402-10.

Claus, Ronald E., and Lisa R. Kindleberger. 2002. "Engaging Substance Abusers after 
Centralized Assessment: Predictors of Treatment Entry and Dropout." Journal of Psychoactive Drugs 34 (1): 25-31.

Dave, Dhaval M., Anca M. Grecu, and Henry Saffer. 2017. "Mandatory Access Prescription Drug Monitoring Programs and Prescription Drug Abuse." NBER Working Paper 23537.

Dave, Dhaval M., and Swati Mukerjee. 2011. "Mental Health Parity Legislation, Cost-Sharing and Substance-Abuse Treatment Admissions." Health Economics 20 (2): 161-83.

Ellyson, Alice, Jevay Grooms, and Alberto Ortega. 2017. "Flipping the Script: the Effect of Prescription Drug Monitoring Programs on Prescribing Behavior." Unpublished.

Grooms, Jevay, and Alberto Ortega. 2018. "Getting with the Program: Medicaid Expansion and Access to Substance Use Treatment." Unpublished.

Haber, Susan G., Galina Khatutsky, and Janet B. Mitchell. 2000. "Covering Uninsured Adults Through Medicaid: Lessons from the Oregon Health Plan." Health Care Finance Review 22 (2): 1-17.

Hamersma, Sarah, and Johanna Catherine Maclean. 2018. "Insurance Expansions and Children's Use of Substance Use Disorder Treatment." NBER Working Paper 24499.

Jones, C. M., M. Campopiano, G. Baldwin, and E. McCance-Katz. 2015. "National and State Treatment Need and Capacity for Opioid Agonist Medication-Assisted Treatment." American Journal of Public Health 105 (8): e55-63.

Maclean, Johanna Catherine, and Brendan Saloner. Forthcoming. "The Effect of Public Insurance Expansions on Substance Use Disorder Treatment: Evidence from the Affordable Care Act." Journal of Policy Analysis and Management.
National Institute of Drug Abuse, Advancing Addiction Science. 2016. "Drugs, Brains, and Behavior: The Science of Addiction." https://www.drugabuse.gov/publications/ drugs-brains-behavior-science-addiction/ introduction.

National Safety Council Statistics Department. 2018. "2017 Estimates Show Vehicle Fatalities Topped 40,000 for Second Straight Year."

Saloner, Brendan, Yaa Akosa Antwi, Johanna Catherine Maclean, and Benjamin Cook. 2018. "Access to Health Insurance and Utilization of Substance Use Disorder Treatment: Evidence from the Affordable Care Act Dependent Coverage Provision." Health Economics 27 (1): 50-75.

Simon, Kosali, Aparna Soni, and John Cawley. 2017. "The Impact of Health Insurance on Preventive Care and Health Behaviors: Evidence from the First Two Years of the ACA Medicaid Expansions." Journal of Public Analysis and Management 36 (2): 390-417.

Substance Abuse and Mental Health Services Administration. 2014. The NSDUH Report: Substance Use and Mental Health Estimates from the 2013 National Survey on Drug Use and Health: Overview of Findings. Rockville, MD.

Substance Abuse and Mental Health Services Administration. 2016. "Co-occurring Disorders." https://www.samhsa.gov/disorders/ co-occurring.

Wen, Hefei, Jason M. Hockenberry, Tyrone F. Borders, and Benjamin G. Druss. 2017. "Impact of Medicaid Expansion on Medicaid-covered Utilization of Buprenorphine for Opioid Use Disorder Treatment." Medical Care 55 (4): 336-41. 